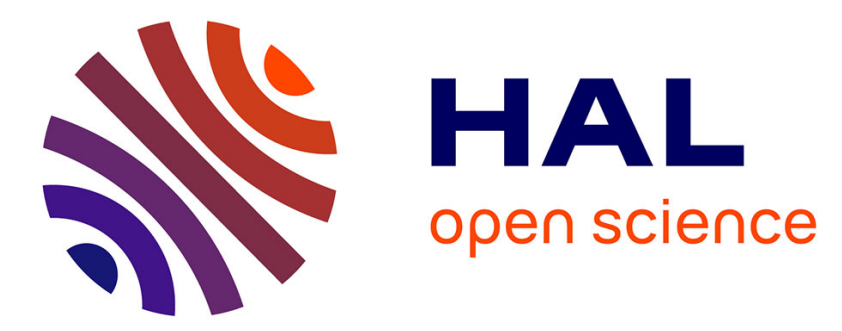

\title{
A pressure-based regularized lattice-Boltzmann method for the simulation of compressible flows
}

G. Farag, S. Zhao, T. Coratger, Pierre Boivin, G. Chiavassa, Pierre Sagaut

\section{To cite this version:}

G. Farag, S. Zhao, T. Coratger, Pierre Boivin, G. Chiavassa, et al.. A pressure-based regularized lattice-Boltzmann method for the simulation of compressible flows. Physics of Fluids, 2020, 32 (6), pp.066106. 10.1063/5.0011839 . hal-02885427

\section{HAL Id: hal-02885427 https://hal.science/hal-02885427}

Submitted on 30 Jun 2020

HAL is a multi-disciplinary open access archive for the deposit and dissemination of scientific research documents, whether they are published or not. The documents may come from teaching and research institutions in France or abroad, or from public or private research centers.
L'archive ouverte pluridisciplinaire HAL, est destinée au dépôt et à la diffusion de documents scientifiques de niveau recherche, publiés ou non, émanant des établissements d'enseignement et de recherche français ou étrangers, des laboratoires publics ou privés. 


\section{A pressure-based regularized Lattice-Boltzmann method for the simulation of}

compressible flows

G. Farag, ${ }^{1}$ S. Zhao, ${ }^{1}$ T. Coratger, ${ }^{1}$ P. Boivin, ${ }^{1}$ a) G. Chiavassa, ${ }^{1}$ and P. Sagaut ${ }^{1}$

Aix Marseille Univ, CNRS, Centrale Marseille, M2P2, Marseille,

France

(Dated: June 17, 2020)

A new pressure-based Lattice-Boltzmann method (HRR- $p$ ) is proposed for the simulation of flows for Mach numbers ranging from 0 to 1.5. Compatible with nearest neighbor lattices (e.g. D3Q19), the model consists of a predictor step comparable to classical athermal Lattice-Boltzmann methods, appended with a fully local and explicit correction step for the pressure. Energy conservation - for which the Hermitian quadrature is not accurate enough on such lattice - is solved via a classical finite volume MUSCL-Hancock scheme based on the entropy equation. The Euler part of the model is then validated for the transport of three canonical modes (vortex, entropy, and acoustic propagation), while its diffusive/viscous properties are assessed via thermal Couette flow simulations. All results match the analytical solutions, with very limited dissipation. Lastly, the robustness of the method is tested in a one dimensional shock tube and a two-dimensional shock - vortex interaction.

a)Electronic mail: pierre.boivin@univ-amu.fr 


\section{INTRODUCTION}

Lattice-Boltzmann methods (LBMs) have received an exponentially growing interest during the last two decades, and are now recognized as a powerful tool for the simulation of flow dynamics ${ }^{1}$. While the Boltzmann equation is a reliable model for compressible flows, with a broader range of validity than the usual compressible Navier-Stokes equations, LBM has historically been mostly developed for low-Mach number flows, and extension to high speed compressible flows is still a challenging topic $\mathrm{c}^{\underline{\underline{2}}} \underline{\underline{4}}$. A key feature of the classical LatticeBoltzmann approach ${ }^{\frac{5}{5}}$ is that the collision term is modeled using a near-equilibrium hypothesis, leading to the use of a relaxation term about the analytical Maxwellian equilibrium solution. For the sake of computational efficiency, the latter is replaced by a truncated polynomial expansion, the order of the expansion being directly related to the capability of the method to account for compressibility effects and thermodynamic mechanisms. The higher the expansion order, the more physics is included, at the price of an increase of the number of discrete distribution functions needed to reconstruct macroscopic quantities by computing the moments of the distribution functions ${ }^{\underline{6}}$.

The main difficulty when designing a LBM for compressible flows is related to the computational efficiency. The key issue is to find a robust and accurate LBM approach with an acceptable computational cost, in terms of both computational time and memory requirement.

As a matter of fact, a fully compressible LBM method can be obtained in a straightforward way by expanding the analytical Maxwellian up to the necessary order and to use the associated required number of distribution function. Unfortunately, such an approach is not tractable for practical flow simulations since it involves at least a 9-th order expansion

of the Maxwellian along with the use of 121 distribution functions for 3D computations ${ }^{\mathbb{T}}$. In such an approach, mass, momentum and energy conservation are recovered at the same time using a single set of distribution functions, corresponding to the coupled approach for compressible Navier-Stokes methods, in which all equations are solved in monolithic way.

To solve this problem, a commonly used approach is to decouple the energy equation from the mass and momentum conservation equation, leading to a segregated approach, according to the classical nomenclature for CFD methods based on Navier-Stokes equations. The expected gain here is to be able to keep using a reduced-order expansion of the Maxwellian 
(compared to the monolithic approach) and a reasonable number of distribution functions, while recovering the full compressible Navier-Stokes equations dynamics. Within the framework of LBM, this leads to the definition of Double Distribution Function (DDF) methods and Hybrid methods $\stackrel{8}{*}$. In the former, the energy equation is solved as the zero-th order of a second set of distribution functions, while in the latter, it is solved using a classical Finite Volume/Finite Difference method for the scalar quantity. Segregated methods raise the issue of the coupling between the block of mass and momentum conservation and the energy equation. Since a restricted Maxwellian expansion is used for the mass/momentum equations, compressibility and thermodynamic effects must be explicitly reintroduced in the associated Lattice-Boltzmann equations ${ }^{9}$. Considering existing DDF and Hybrid methods for high-speed compressible flows, the main coupling ways are: i) to keep using a low-Mach athermal collision model and to enforce the physical pressure gradient as a forcing term, or ii) to use a low-Mach thermal collision model supplemented by some forcing correction terms. These choices are observed to have a deep impact on the features of the resulting numerical method in terms of accuracy and robustness. The first approach leads to some numerical stability problems near discontinuities, while the second is more robust but still necessitates some additional stabilization techniques ${ }^{10}$. A weakness of the second approach is that the positivity of the expanded equilibrium function is no longer guaranteed 2 and necessitates the definition of a case-dependent reference temperature. $\operatorname{In}^{4} \underline{10}-14$ a Hybrid density-based LBM based on the second approach has been recently proposed (referred to as HRR- $\rho$ hereafter), with successful application to thermal compressible flows in both subsonic and supersonic regimes on a regular D3Q19 lattice (i.e. with 19 discrete velocities for $3 \mathrm{D}$ flows). The key features of this method are i) the use of an dynamic hybrid recursive regularized (HHR) collision mode ${ }^{15}$ supplemented by ad hoc correction terms and ii) solving an evolution equation for entropy written in non-conservative form. The stability is explicitly controlled by the dynamic parameter in the collision kernel, which is shown $\frac{16}{}$ to damp unphysical ghost modes $\frac{17}{7}$, but also to introduce some artificial bulk viscosity $\underline{16}, \underline{18,19}$.

The goal of the present paper is to propose new improvements of this method, aiming at limiting the amount of bulk viscosity while increasing the stability and robustness of the method. To this end, two major changes are introduced in the previous version of the method. In the first novelty, the collision model is changed to get a pressure-based approach $^{20}-24$ instead of the previous density-based HRR- $\rho$, leading to an improved con- 
trol of spurious pressure waves without addition of bulk viscosity while keeping the same hybrid recursive regularization technique. In the second novelty, the coupling between the mass/momentum block and the entropy equation is now performed using a predictorcorrector approach, that allows for the use of an athermal collision kernel, eliminating the sensitivity to the definition of a case-dependent reference temperature. These new elements yield the definition of a new segregated, pressure-based hybrid LBM (referred to as HRR- $p$ ) equipped with a HRR collision model and an entropy equation.

Segregated pressure-based LBMs have been investigated by many authors during the last two decades, only for low-Mach flows, e.g. low-Mach thermal flows with variable properties $\frac{25}{}$, low-Mach combustion $\underline{\underline{26}} \underline{\underline{29}}$, low-Mach multiphase flows $\underline{20} \underline{\underline{24}}, \underline{30} \underline{-38}$ including phase change and thermal phase change $\underline{39}^{\underline{43}}$. In almost all cases, the DDF approach was used to solve the additional equation (e.g. phase index, temperature ...); only very few authors used a hybrid approach with a Finite Difference method for the scalar temperature/energy equation $\underline{26}, 27,39,43$. To the knowledge of the authors, the present HRR- $p$ method is the first one dealing with segregated pressure-based method for high-speed compressible flows in both subsonic and supersonic regimes, using a hybrid finite-difference-based approach to solve the entropy equation.

The paper is organized as follows. The set of macroscopic equations associated to the present method is presented in Section II. The general structure of the present approach is discussed in Section ЩIA, while the details of the implementation are given in Section IIIB. Numerical results obtained with the new method are displayed in Section IV, followed by the conclusions in Section $\mathrm{V}$,

\section{MACROSCOPIC GOVERNING EQUATIONS: CONTINUOUS FORMULATION}

In the present new hybrid model, a pressure-based predictor corrector Lattice-Boltzmann solver is used for mass and momentum equations

$$
\begin{gathered}
\frac{\partial \rho}{\partial t}+\frac{\partial \rho u_{\beta}}{\partial x_{\beta}}=0 \\
\frac{\partial \rho u_{\alpha}}{\partial t}+\frac{\partial \rho u_{\alpha} u_{\beta}+\delta_{\alpha \beta} p-\Pi_{\alpha \beta}}{\partial x_{\beta}}=0
\end{gathered}
$$


along with a finite difference scheme for the entropy equation

$$
\frac{\partial s}{\partial t}+u_{\beta} \frac{\partial s}{\partial x_{\beta}}=\frac{1}{\rho T}\left[\Pi_{\alpha \beta} \frac{\partial u_{\alpha}}{\partial x_{\beta}}-\frac{\partial q_{\beta}}{\partial x_{\beta}}\right],
$$

where the stress tensor $\Pi_{\alpha \beta}$ and the heat flux $q_{\alpha}$ have been defined as

$$
\Pi_{\alpha \beta}=\mu\left(\frac{\partial u_{\alpha}}{\partial x_{\beta}}+\frac{\partial u_{\beta}}{\partial x_{\alpha}}-\delta_{\alpha \beta} \frac{2}{3} \frac{\partial u_{\gamma}}{\partial x_{\gamma}}\right), q_{\alpha}=-\lambda \frac{\partial T}{\partial x_{\alpha}},
$$

with $\mu$ the dynamic viscosity and $\lambda$ the heat conductivity. Thermodynamic closure follows the usual perfect gas assumption

$$
p=\rho r T, \quad s=C_{v} \ln \frac{p}{\rho^{\gamma}}
$$

where $C_{v}$ is the specific heat capacity, $r=\frac{R}{W}$ is the specific gas constant, $R$ is the universal gas constant, $W$ is the molecular weight and $\gamma=C_{p} / C_{v}$ is the adiabatic exponent. In the following, we introduce the thermodynamic quantity $\theta$

$$
\theta=\frac{r T}{r T_{0}}=\frac{r T}{c_{s}^{2}}
$$

as the ratio of the thermodynamic perfect gas pressure to the classical athermal LBM pressure $^{9} p=\rho c_{s}^{2} . \quad c_{s}$ is a constant characteristic lattice velocity ${ }^{9}$, not to be confused with the temperature-dependent physical sound speed $c^{2}=\sqrt{\gamma r T}$.

\section{NUMERICAL METHOD: A SEGREGATED PRESSURE-BASED LBM PREDICTOR-CORRECTOR APPROACH}

\section{A. LBM-based predictor-corrector approach for compressible flows}

The present algorithm is based on a predictor-corrector approach, in which the weakly compressible solution of the predictor step is corrected to recover a fully compressible solution. The efficiency of such a method, as all similar methods developed within the NavierStokes framework for deriving a compressible flow solver starting from an incompressible solver, depends on the robustness and numerical efficiency of the predictor step $\underline{44} \underline{46}$. According to the classification used for Navier-Stokes-based methods ${ }^{47-50}$, the present approach is a segregated, pressure-based method, in which both the predictor and the corrector steps rely on purely explicit schemes, without any subiteration process. As a compromise between robustness and efficiency, it is chosen here to use a predictor based on the Artificial 
Compressibility Method pioneered by Chorin ${ }^{51,52}$, which is also close to the limited compressibility approach introduced by Hirt ${ }^{53}$ to extend elliptic solvers for incompressible flows. The artificial compressibility method has been widely used and improved during the last 50 years, e.g. $\underline{54} \underline{57}$. The similarity between LBMs for low-Mach athermal flows and Artificial Compressibility Method was analyzed in ${ }^{58}$, and further exploited in the definition of improved methods for low-Mach thermal flows, e.g. ${ }^{59}$.

The proposed method can be written in semi-discrete form as follows for macroscopic variables :

1. Predictor: starting from solution at time step $n$ : $\left(\boldsymbol{u}^{n}, \rho^{n}, T^{n}, p^{n}, s^{n}, \Pi^{n}, \boldsymbol{q}^{n}\right)$, solve

$$
\begin{cases}\left(\frac{p^{*}-p^{n}}{c_{s}^{2} \Delta t}\right) & =-\nabla \cdot\left(\rho^{n} \boldsymbol{u}^{n}\right) \\ \left(\frac{\rho^{n+1} \boldsymbol{u}^{n+1}-\rho^{n} \boldsymbol{u}^{n}}{\Delta t}\right) & =-\nabla \cdot\left(\rho^{n} \boldsymbol{u}^{n} \boldsymbol{u}^{n}\right)-\nabla \cdot p^{n}+\nabla \cdot \Pi^{n}\end{cases}
$$

to get $\rho^{n+1} \boldsymbol{u}^{n+1}$ and $p^{*}$.

2. Corrector: solve the entropy equation

$$
\left(\frac{s^{n+1}-s^{n}}{\Delta t}\right)=-\boldsymbol{u}^{n} \cdot \nabla s^{n}+\frac{1}{\rho^{n} T^{n}}\left[\Pi^{n} \nabla \boldsymbol{u}^{n}-\nabla \cdot \boldsymbol{q}^{n}\right]
$$

and correct the density, pressure and temperature following

$$
\begin{aligned}
\rho^{n+1} & =\rho^{n}+\Delta t\left(\frac{\partial \rho}{\partial t}\right)^{n} \\
& =\rho^{n}-\Delta t(\nabla \cdot(\rho \boldsymbol{u}))^{n}=\rho^{n}+\frac{1}{c_{s}^{2}}\left(p^{*}-p^{n}\right)
\end{aligned}
$$

and then one has

$$
p^{n+1}=\left(\rho^{n+1}\right)^{\gamma} \mathrm{e}^{s^{n+1} / C_{v}}, \quad T^{n+1}=p^{n+1} / r \rho^{n+1}
$$

from which $\Pi^{n+1}$ and $\boldsymbol{q}^{n+1}$ can be computed in a straightforward way. 
For the sake of efficiency and getting control of spurious pressure waves, the predictor step is implemented using a pressure-based Lattice-Boltzmann approach as proposed by ${ }^{21}, 33,60$ equipped with the HRR collision model ${ }^{4,15}$. Pressure-based collision kernels have been initially proposed to handle low-Mach flows with large density variations (e.g. multiphase interfacial flows), exhibiting much better robustness and accuracy than classical single relaxation time density-based LBMs for such flows, thanks to their explicit control of pressure oscillations in flows exhibiting large density variations. While pressure-based and density-based approaches share the same equivalent macroscopic equations in the lowMach athermal limit, computing the pressure directly via an evolution equation instead of calculating it by the equation of state is known to reduce spurious pressure oscillations, as pointed out in $\underline{61,62}$ for multifluid Navier-Stokes methods and in $\underline{26,27,60}$ for LBMs. Even in the single-phase monofluid case, high-speed high-Mach flows exhibit large density variations, e.g. in the presence of discontinuities like shock waves and slip lines, and also in the presence of strong travelling pressure waves. Therefore, an improved control of pressure oscillations will be beneficial for this class of flows. It is worth noting that the pressure evolution equation obtained in the predictor step is not valid for high Mach number flows. As a matter of fact, it corresponds to the low-Mach athermal limit of the full compressible equation $\underline{57,60,62,63}$, therefore requiring a correction step to recover the full compressible flow physics.

In order to further enhance the stability and the accuracy of the method, it is decided here to use an advanced collision kernel with accurate ghost mode damping properties with an explicit control of the artificial hyperviscosity, namely the HRR scheme, which has been recently observed to be very efficient for a large class of flows, ranging from low-Mach athermal flows $\frac{15,64}{6}$ to supersonic flows $10,11,14$, including reactive flows $\frac{12,13}{13}$ and saturated humid gas flows ${ }^{4}$, while keeping a mere regular D3Q19 lattice in three dimensions $\stackrel{40}{-14}$. Linearized spectral analysis conducted in ${ }^{16}$ also revealed that it has a great capability of damping spurious ghost modes $\frac{17}{}$.

It is chosen to use an hybrid approach, in which the entropy equation is solved using a classical finite-difference scheme to optimize the efficiency of the method in terms of the computational cost and memory storage, as done in $\underline{\underline{4}} \underline{\underline{10}} \underline{\underline{14}}$. 


\section{B. Lattice-Boltzmann implementation}

We now detail the implementation of the previous method within the LBM framework, involving the stream-collide splitting $\underline{\underline{65}}$ between the advection and the collision steps. The present pressure-based method relies on a D3Q19 lattice with distribution function $f_{i}$ such that

$$
\left\{\begin{array}{l}
\sum_{i} f_{i}^{e q}=\rho \theta=p / c_{s}^{2}, \\
\sum_{i} c_{i, \alpha} f_{i}^{e q}=\rho u_{\alpha}, \\
\sum_{i} c_{i, \alpha} c_{i, \beta} f_{i}^{e q}=\rho u_{\alpha} u_{\beta}+\rho \theta c_{s}^{2} \delta_{\alpha \beta},
\end{array}\right.
$$

which obeys the classical single relaxation time evolution equation (in which possible additional forcing terms have been omitted for the sake of simplicity)

$$
\frac{\partial f_{i}}{\partial t}+\xi_{\alpha} \frac{\partial f_{i}}{\partial x_{\alpha}}=-\frac{1}{\tau}\left(f_{i}-f_{i}^{\text {eq, }, 19 r}\right),
$$

where the collision step is based on the following modified pressure-based third order equilibrium distribution

$$
\begin{aligned}
& f_{i}^{\text {eq, } 19 r}=\omega\left\{\rho \theta+\frac{\mathcal{H}_{i \alpha}^{(1)}}{c_{s}^{2}} \rho u_{\alpha}+\frac{\mathcal{H}_{i \alpha \beta}^{(2)}}{2 c_{s}^{4}} \rho u_{\alpha} u_{\beta}+\frac{1}{6 c_{s}^{6}}[\right. \\
& \quad+3\left(\mathcal{H}_{i, x x y}^{(3)}+\mathcal{H}_{i, y z z}^{(3)}\right)\left(\rho u_{x} u_{x} u_{y}+\rho u_{y} u_{z} u_{z}\right) \\
& \quad+\left(\mathcal{H}_{i, x x y}^{(3)}-\mathcal{H}_{i, y z z}^{(3)}\right)\left(\rho u_{x} u_{x} u_{y}-\rho u_{y} u_{z} u_{z}\right) \\
& +3\left(\mathcal{H}_{i, x z z}^{(3)}+\mathcal{H}_{i, x y y}^{(3)}\right)\left(\rho u_{x} u_{z} u_{z}+\rho u_{x} u_{y} u_{y}\right) \\
& \quad+\left(\mathcal{H}_{i, x z z}^{(3)}-\mathcal{H}_{i, x y y}^{(3)}\right)\left(\rho u_{x} u_{z} u_{z}-\rho u_{x} u_{y} u_{y}\right) \\
& +3\left(\mathcal{H}_{i, y y z}^{(3)}+\mathcal{H}_{i, x x z}^{(3)}\right)\left(\rho u_{y} u_{y} u_{z}+\rho u_{x} u_{x} u_{z}\right) \\
& \left.\left.+\left(\mathcal{H}_{i, y y z}^{(3)}-\mathcal{H}_{i, x x z}^{(3)}\right)\left(\rho u_{y} u_{y} u_{z}-\rho u_{x} u_{x} u_{z}\right)\right]\right\} .
\end{aligned}
$$

Here, the equilibrium function is expressed in the D3Q19 rotational symmetry basis of Gauss-Hermite polynomials $\frac{15}{\underline{15}}$

$$
\begin{gathered}
\mathcal{H}_{i}^{(0)}=1, \quad \mathcal{H}_{i \alpha}^{(1)}=c_{i \alpha}, \\
\mathcal{H}_{i \alpha \beta}^{(2)}=c_{i \alpha} c_{i \beta}-c_{s}^{2} \delta_{\alpha \beta}, \\
\mathcal{H}_{i \alpha \beta \gamma}^{(3)}=c_{i \alpha} c_{i \beta} c_{i \gamma}-c_{s}^{2}\left[c_{i \alpha} \delta_{\beta \gamma}+c_{i \beta} \delta_{\gamma \alpha}+c_{i \gamma} \delta_{\alpha \beta}\right],
\end{gathered}
$$

to reduce the defect of the third-order moment $\frac{15}{}$.

Note that the previous HRR- $\rho$ model proposed in $\underline{\underline{66}}$ encompassed the thermal effect inside the second and third order moments of the density distribution, while this new model can 
be seen as an athermal equilibrium with a modified zeroth order moment $\rho \theta \equiv \frac{p}{c_{s}^{2}}$. Due to this change in the zeroth order, the corresponding conservation equation recovered from Eq. (12) now reads

$$
\frac{\partial \rho \theta}{\partial t}+\frac{\partial \rho u_{\alpha}}{\partial x_{\alpha}}=0
$$

leading to a pressure equation in the low Mach limit ${ }^{58}$. The momentum conservation equation is not directly impacted and remains unchanged as first and second order moments of $f_{i}^{\text {eq, } 19 r}$ are still physically consistent. In particular, the first order moment of Eq. (12) yields

$$
\frac{\partial \rho u_{\alpha}}{\partial t}+\frac{\partial \rho u_{\alpha} u_{\beta}}{\partial x_{\beta}}=-\frac{\partial \rho \theta c_{s}^{2}}{\partial x_{\alpha}}-\frac{\partial a_{\alpha \beta}^{\text {neq }}}{\partial x_{\beta}}
$$

where $a_{\alpha \beta}^{\text {neq }}$ is the second order non-equilibrium tensor. At this stage, we only have a low Mach thermal solver, which defines our predictor. In order to recover a fully compressible solution, it is mandatory to correct both macroscopic moments and density distributions during the following modified stream and collide algorithm :

1. Initialization. The algorithm starts with a collide step. The first post-collide population $f_{i}^{\text {col }}$ is initialized at each grid point as

$$
f_{i}^{\mathrm{col}}\left(t_{0}, \boldsymbol{x}\right)=f_{i}^{\mathrm{eq}}\left(t_{0}, \boldsymbol{x}\right)
$$

with $f_{i}^{\text {eq, } 19 r}$ being the equilibrium distribution evaluated from density, velocity and temperature at initial time step.

2. Streaming. With knowing the collide population at time step $t$, an intermediate population $f_{i}^{*}$ is obtained by a streaming step from neighbors

$$
f_{i}^{*}(t+\Delta t, \boldsymbol{x})=f_{i}^{\mathrm{col}}\left(t, \boldsymbol{x}-\boldsymbol{c}_{\boldsymbol{i}} \Delta t\right)
$$

3. Regularized collision. The collide population at time step $t+\Delta t$ is achieved by

i) Updating macroscopic variables. The density and velocity fields at the next time level are obtained from the post-streaming populations $f_{i}^{*}$ as

$$
\begin{gathered}
\rho(t+\Delta t, \boldsymbol{x})=\sum_{i} f_{i}^{*}(t+\Delta t, \boldsymbol{x})+\rho(t, \boldsymbol{x})[1-\theta(t, \boldsymbol{x})] \\
\left(\rho u_{\alpha}\right)(t+\Delta t, \boldsymbol{x})=\sum_{i} \boldsymbol{c}_{\boldsymbol{i}_{\alpha}} f_{i}^{*}(t+\Delta t, \boldsymbol{x})
\end{gathered}
$$

Along with this step, the scalar equation Eq. (8) for the entropy $s$ is advanced in time using Finite Difference discretization. Knowing $s(t+\Delta t, \boldsymbol{x})$ and $\rho(t+\Delta t, \boldsymbol{x})$, it is possible to get $\theta(t+\Delta t, \boldsymbol{x})$ by use of the thermodynamic closure Eq. (5) . 
ii) Constructing the off-equilibrium tensors. The non-equilibrium part of $f_{i}^{*}$ is defined by

$$
\begin{aligned}
f_{i}^{\text {neq* }}(t+\Delta t, \boldsymbol{x}) & =f_{i}^{*}(t+\Delta t, \boldsymbol{x})-f_{i}^{\mathrm{eq}}(t+\Delta t, \boldsymbol{x}) \\
& +\frac{1}{2} F_{i}^{E}(t+\Delta t, \boldsymbol{x})
\end{aligned}
$$

where $f_{i}^{\text {eq }}$ is calculated from $\rho, u$ and $\theta$ at time level $t+\Delta t$ using Eq. (13), and $F_{i}^{E}$ is a forcing term explicited in Eq. (A5) of Appendix A. It is worth noting that at this stage, all the variables needed for the equilibrium distribution $f_{i}^{\text {eq }}$ and forcing term $F_{i}^{E}$ are available thanks to the entropy update in the last substep. The second order non-equilibrium tensor is estimated by

$$
a_{\alpha \beta}^{\mathrm{neq}}(t+\Delta t, \boldsymbol{x})=\mathcal{H}_{i, \alpha \beta}^{(2)}\left(\boldsymbol{c}_{\boldsymbol{i}}\right) f_{i}^{\text {neq* }}(t+\Delta t, \boldsymbol{x})
$$

The hybrid recursive collision model ${ }^{15}$ can then be embedded to achieve extra numerical stability, as well as enforcing a traceless Lattice-Boltzmann tensor by removing its trace. The resulted viscous stress tensor then reads

$$
\tilde{a}_{\alpha \beta}^{\text {neq }} \equiv \sigma\left[a_{\alpha \beta}^{\text {neq }}-\frac{\delta_{\alpha \beta}}{3} a_{\gamma \gamma}^{\text {neq }}\right]+(1-\sigma) a_{\alpha \beta}^{\text {neq,FD }}
$$

where $a_{\alpha \beta}^{\text {neq,FD }}$ is evaluated directly from a second order centered finite difference (FD) scheme of

$$
a_{\alpha \beta}^{\text {neq }}=-\rho c_{s}^{2} \bar{\tau}\left(u_{\alpha, \beta}+u_{\beta, \alpha}-\frac{2}{3} u_{\gamma, \gamma} \delta_{\alpha \beta}\right),
$$

where $\bar{\tau}=\tau+\Delta t / 2$ is the relaxation time and

$$
\tau=\frac{\mu}{\rho c_{s}^{2}}
$$

iii) Collision. The post-collide population at time step $t+\Delta t$ is calculated as

$$
\begin{gathered}
f_{i}^{\mathrm{col}}(t+\Delta t, \boldsymbol{x})=f_{i}^{\mathrm{eq}}(t+\Delta t, \boldsymbol{x}) \\
+\left(1-\frac{1}{\bar{\tau}}\right) f_{i}^{\mathrm{neq}}(t+\Delta t, \boldsymbol{x})+\frac{1}{2} F_{i}^{E}(t+\Delta t, \boldsymbol{x})
\end{gathered}
$$

in which the forcing term $F_{i}^{E}$ is added to recover an accurate non-equilibrium tensor as detailed in Appendix $\mathrm{A}$, and the non-equilibrium regularized population $f_{i}^{\text {neq }}$ is evaluated as

$$
f_{i}^{\text {neq }} \equiv \omega_{i}\left[\frac{1}{2 c_{s}^{4}} \mathcal{H}_{i, \alpha \beta}^{(2)} \tilde{a}_{\alpha \beta}^{\text {neq }}+\frac{1}{6 c_{s}^{6}} \mathcal{H}_{i, \gamma}^{(3 r)} a_{\gamma}^{(3 r), \text { neq }}\right],
$$


with the third order off-equilibrium $a_{\gamma}^{(3 r) \text {,neq }}$ recursively derived as detailed in Appendix B.

4. Repeat streaming and collision steps until the last time step.

In the above algorithm, Eq. (19) is the correction to recover the mass equation. If no special treatment is applied, one should update $(\rho \theta) \equiv \sum_{i} f_{i}^{*}$ at the next time step for the macroscopic variable at zeroth Hermite order instead of $\rho$, i.e.

$$
(\rho \theta)^{*}(t+\Delta t, \boldsymbol{x})=\sum_{i} f_{i}^{*}(t+\Delta t, \boldsymbol{x})
$$

Reminding that $\rho \theta=\frac{p}{c_{s}^{2}}$, it leads to the pressure Eq. (15) in the low Mach limit 58 . However, slightly changing Eq. (28), the following relationship can be established

$$
\begin{aligned}
(\rho \theta)^{*}(t+\Delta t, \boldsymbol{x})-(\rho \theta)(t, \boldsymbol{x}) & =\sum_{i} f_{i}^{*}(t+\Delta t, \boldsymbol{x})-(\rho \theta)(t, \boldsymbol{x}) \\
& \approx-\frac{\partial \rho u_{\alpha}}{\partial x_{\alpha}} \Delta t
\end{aligned}
$$

according to Eq. (15). This pseudo mass flux can be used to update the density as

$$
\begin{aligned}
\rho(t+\Delta t, \boldsymbol{x}) & \approx-\frac{\partial \rho u_{\alpha}}{\partial x_{\alpha}} \Delta t+\rho(t, \boldsymbol{x}) \\
& =(\rho \theta)^{*}(t+\Delta t, \boldsymbol{x})-(\rho \theta)(t, \boldsymbol{x})+\rho(t, \boldsymbol{x}) \\
& =\sum_{i} f_{i}^{*}(t+\Delta t, \boldsymbol{x})-\rho(t, \boldsymbol{x}) \theta(t, \boldsymbol{x})+\rho(t, \boldsymbol{x})
\end{aligned}
$$

which leads to Eq. (19).

Unfortunately, by doing so, the second order moment of the population is altered. In order to obtain a stress tensor like in Eq. (A1), the correction term given in Eq. (A3) must be injected into the forcing term.

One favorable feature of the HRR- $p$ scheme is that the total mass and momentum are numerically conserved. For an infinite (or periodic) spatial domain, it is easy to demonstrate that the total mass at the next time step is 


$$
\begin{array}{rlrl}
\int \rho(t+\Delta t, \boldsymbol{x}) d V & \doteq & \sum_{\boldsymbol{x}}\left[\sum_{i} f_{i}^{*}(t+\Delta t, \boldsymbol{x})-\rho(t, \boldsymbol{x}) \theta(t, \boldsymbol{x})+\rho(t, \boldsymbol{x})\right] \\
& = & \sum_{\boldsymbol{x}} \sum_{i} f_{i}^{\mathrm{col}}\left(t, \boldsymbol{x}-\boldsymbol{c}_{\boldsymbol{i}} \Delta t\right)+\sum_{\boldsymbol{x}}[\rho(t, \boldsymbol{x})(1-\theta(t, \boldsymbol{x}))] \\
& = & \sum_{\boldsymbol{x}} \sum_{i} f_{i}^{c o l}(t, \boldsymbol{x})+\sum_{\boldsymbol{x}}[\rho(t, \boldsymbol{x})(1-\theta(t, \boldsymbol{x}))] \\
& =\sum_{\boldsymbol{x}} \sum_{i}[f_{i}^{\mathrm{eq}}(t, \boldsymbol{x})+\underbrace{\left.\left(1-\frac{1}{\tau}\right) f_{i}^{\mathrm{neq}}(t, \boldsymbol{x})+F_{i}^{E}\right]}_{0}+\sum_{\boldsymbol{x}}[\rho(t, \boldsymbol{x})(1-\theta(t, \boldsymbol{x}))] \\
& = \\
& = & \sum_{\boldsymbol{x}} \sum_{i} f_{i}^{\mathrm{eq}}(t, \boldsymbol{x})+\sum_{\boldsymbol{x}}[\rho(t, \boldsymbol{x})(1-\theta(t, \boldsymbol{x}))] \\
& \doteq & & \int \rho(t, \boldsymbol{x}) \theta(t, \boldsymbol{x})+\sum_{\boldsymbol{x}}[\rho(t, \boldsymbol{x})(1-\theta(t, \boldsymbol{x}))]
\end{array}
$$

thus is conserved during time marching. Following the same manner, it can be shown that the total momentum is also conserved.

\section{Finite difference solver for the entropy equation}

Lastly, let us address the finite difference discretization of the entropy equation, as required in updating the entropy following Eq. (8) during the step i) of the regularized collision.

The convective flux of Eq. (8) is computed by a MUSCL-Hancock method (see Appendix C for details).

Thermal conduction and viscous heat are computed through a simple second order centered finite difference.

\section{RESULTS}

In this section, the proposed HRR- $p$ is assessed on different configurations and compared to reference solutions that can be either analytical solutions or numerical solutions obtained by high-order Navier-Stokes Fourier solvers. The validations are aimed at demonstrating the ability of the present model to accurately reproduce fully compressible effects for a wide range of physical parameters, including

- convection tests at Mach=(0.5, 1, 1.5) for the three fundamental Euler modes: vorticity (Sec. IVA), entropy (Sec. IVB) and acoustic (Sec. IVC). These tests validate the behavior of the model in solving accurately the Euler part of the conservation Eqs. (113), and show highly non-dissipative and non-dispersive properties. 
- Couette thermal flow tests in Sec. IVD, This Section validates the accuracy of the model regarding for the viscous and heat transfer terms of the conservation Eqs. (173), for a wide range of $(M a, \operatorname{Pr}, \gamma)$ numbers.

- validations in the presence of shocks. They include one-dimensional shock tube (Sec. IVE), and shock-vortex interaction (Sec. IVF).

All inviscid simulations are carried out setting the dynamic viscosity to $\mu=10^{-15}$.

The classical definition for the acoustic $C F L$ number

$$
C F L=\frac{\left|u_{0}\right|+c}{\Delta x / \Delta t}
$$

is adopted throughout this Section.

\section{A. Isentropic vortex advection}

The first test case is the usual inviscid and isentropic vortex advected by a mean uniform flow in a fully periodic domain. The analytical solution is a frozen pattern simply advected by the mean flow over time.

The $[0,10] \times[0,10]$ physical domain is discretized by a $200 \times 200$ mesh. The isentropic vortex is initialized at its center and defined as

$$
\begin{aligned}
& \rho=\left[1-\frac{(\gamma-1)}{2} M_{v}^{2} e^{1-r^{2} / R^{2}}\right]^{\frac{1}{\gamma-1}}, \quad p=\rho^{\gamma}, \\
& u=u_{0}-M_{v} \sqrt{\gamma} e^{\left(1-r^{2} / R^{2}\right) / 2}\left(y-y_{c}\right), \\
& v=M_{v} \sqrt{\gamma} e^{\left(1-r^{2} / R^{2}\right) / 2}\left(x-x_{c}\right),
\end{aligned}
$$

with $r=\sqrt{\left(x-x_{c}\right)^{2}+\left(y-y_{c}\right)^{2}}$. The characteristic radius of the spot is set to $R=1$, the free stream flow to $u_{0}=M a \sqrt{\gamma}, T_{0}=1, \rho=1, \gamma=1.4$ and the strength of the vortex to $M_{v}=\frac{1}{4 \pi \sqrt{\gamma}}$, following $\underline{\underline{6}}$. The time-step is fixed to $\Delta t=0.001725$, corresponding to an acoustic $C F L \approx 0.1$ for the $M a=1.5$ case. The value of the HRR weighting parameter is $\sigma=1$ leading to a stress tensor $100 \%$ evaluated by the LBM. The vortex is then advected on a distance of $200 R$ corresponding to 20 flow-through-time (FTT). The initial and final density maps with identical colorbars are reported for different values of the Mach number in Fig. 1. As expected, the shape of the vortex is perfectly preserved after $20 \mathrm{FFT}$, regardless of the Mach value (0.5, 1 and 1.5). 


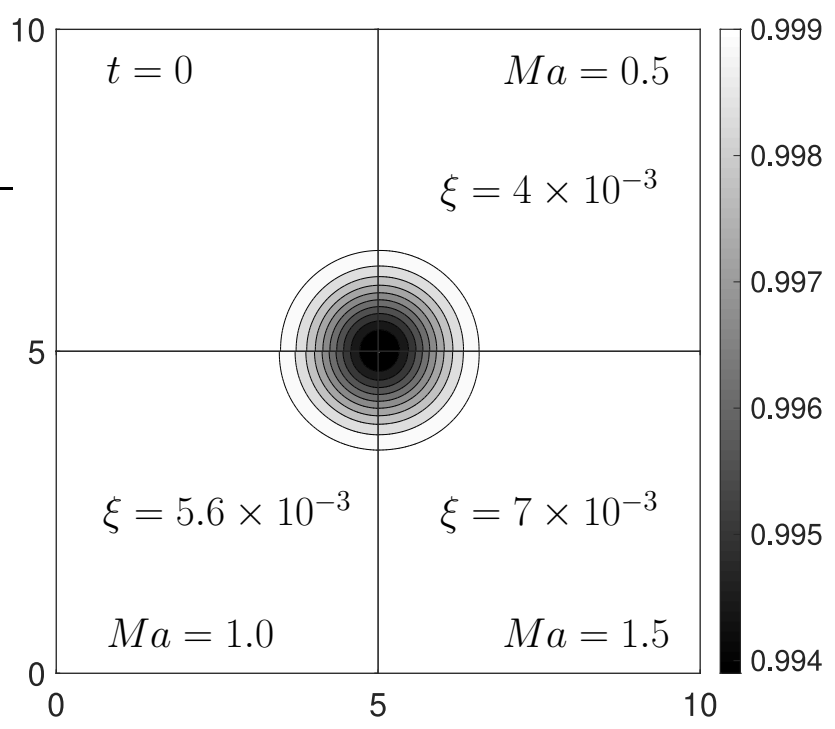

Figure 1: Density fields for the isentropic vortex convection: initial/analytical profile (top left), and solution after $20 F T T$ for $M a=(0.5,1.0,1.5)$. The dissipation $\xi$ is reported in each case.

For each Mach number, the dissipation, defined as $\xi=\frac{\min \rho(20 F T T)-\min \rho(t=0)}{1-\min \rho(t=0)}$ is also reported in Fig. 1, showing that less than 1\% of the initial amplitude was lost after 20 FTT. Note that use of $\sigma=1$ was possible in these simulations, whereas maximum values of $\sigma$ close to 0.7 where achieved with the HRR- $\rho$ formulation $\underline{10}$. This translates to a decrease in dissipation $\xi$ by about an order of magnitude compared with the HRR- $\rho$ model ${ }^{10}$.

The proposed HRR- $p$ method is then able to compute accurately the advection of a radial flow over a long time without introducing spatial distortion nor spurious dissipation, despite a relatively low spatial resolution.

\section{B. Entropy spot advection}

The convected entropy spot is a benchmark of particular interest for the present model as it is expected for the numerical solution to be mainly dependent on the finite difference part of the solver used for the entropy equation. The LBM is known to exhibit a low numerical dissipation behavior $\frac{67}{}$, it is then important to check that the $\mathrm{FV}$ part of the scheme does not deteriorate this property. A good way to verify it is to convect a pure entropy spot over 


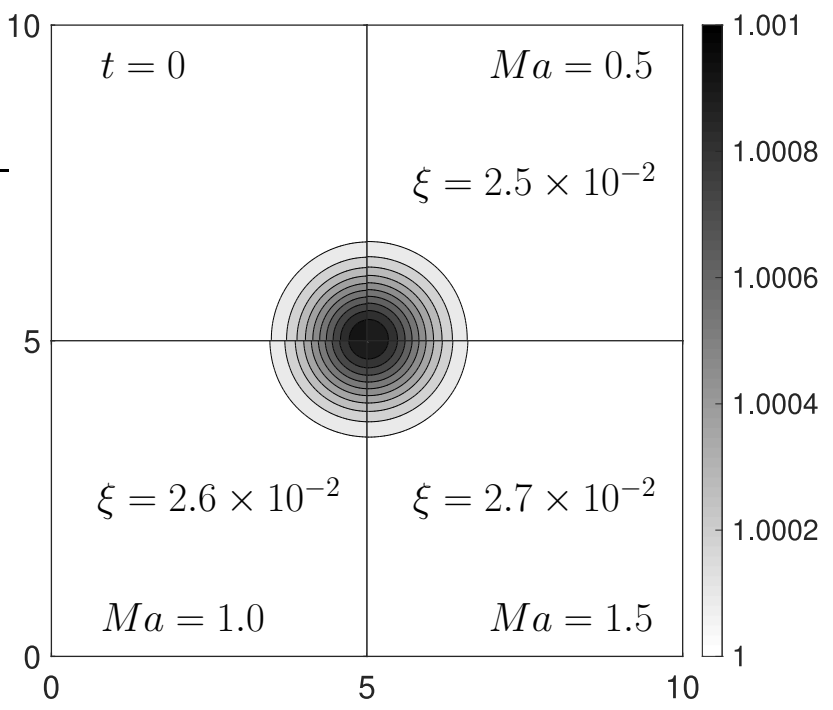

Figure 2: Density fields for the entropy spot convection: initial/analytical profile (top left), and solution after $20 F T T$ for $M a=(0.5,1.0,1.5)$.

a long distance. The flow is then initialized as

$$
\begin{aligned}
& \rho=\rho_{0}\left(1+\epsilon e^{-r^{2} / R^{2}}\right), \\
& T=T_{0}\left(1-\epsilon e^{-r^{2} / R^{2}}\right), \\
& u=u_{0}, \quad v=0,
\end{aligned}
$$

with a sufficiently low value of $\epsilon=10^{-3}$ ensuring that the $u \pm c$ acoustic modes are not triggered $\underline{68}$. All the other parameters remain unchanged compared to the isentropic vortex aside the dissipation which now reads as $\xi=\frac{\max \rho(20 F T T)-\max \rho(t=0)}{1-\max \rho(t=0)}$. Initial and final solutions of the advected entropy spot can be seen on Fig. 2, leading to the conclusion that the shape of the entropy spot is well preserved and that less than $3 \%$ of the maximum amplitude was lost over the whole simulation.

\section{Acoustic wave propagation}

We compute in this part the propagation of a pure acoustic wave over a long distance. The wave is not simply advected by the mean flow, it is propagated with a $u \pm c$ velocity, which leads to a complex pattern when initialized in a $2 \mathrm{D}$ domain. It was then chosen to test the acoustic decay in a $1 \mathrm{D}$ periodic simulation with a $[0,10]$ physical domain discretized 


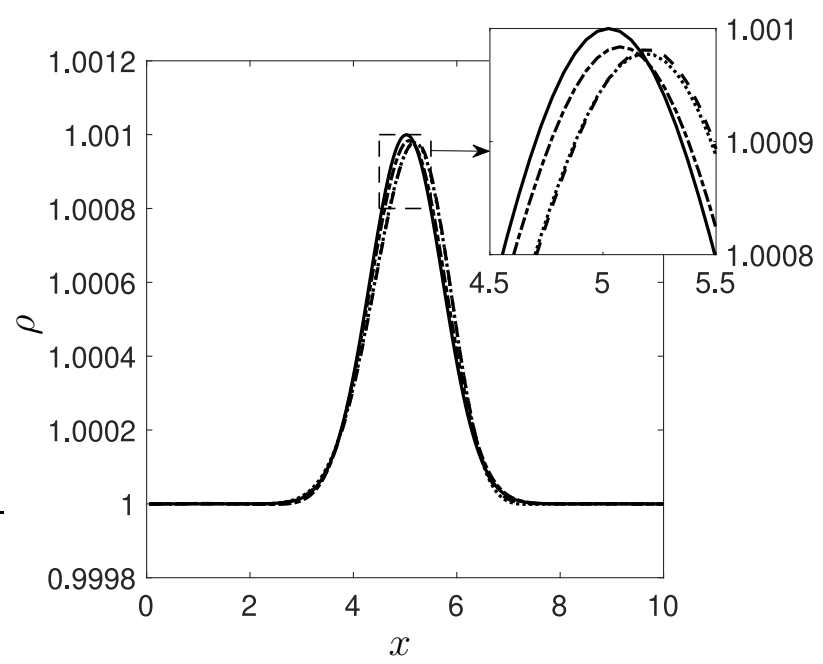

Figure 3: Density fields for the acoustic propagation $(u+c$ mode): initial/analytical profile (solid line), and solution after 20FTT at $M a=0.5$ (dashed), $M a=1.0$ (dotted) and

$$
M a=1.5 \text { (dot-dashed). }
$$

by 200 points. The acoustic wave is initialized as

$$
\begin{aligned}
& \rho=\rho_{0}\left(1+\epsilon e^{-r^{2} / R^{2}}\right) \\
& T=T_{0}\left(1+(\gamma-1) \epsilon e^{-r^{2} / R^{2}}\right), \\
& u=u_{0}+c_{0} \epsilon e^{-r^{2} / R^{2}} \\
& v=0
\end{aligned}
$$

with $r=\left(x-x_{c}\right)$ and $\epsilon=10^{-3}$. Analytical and numerical solutions are plotted on Fig. 3 ,

A good agreement with the analytical solution is observed, with a very low numerical dissipation $\xi$ of respectively $1.9 \times 10^{-2}, 2.2 \times 10^{-2}$ and $1.6 \times 10^{-2}$ for subsonic, sonic and supersonic cases.

\section{Thermal Couette Flow}

Having validated the HRR- $p$ model the Euler part of the conservation equations (1-3) through convection tests, let us now validate the shear stress and heat transfer balance through an analysis of thermal Couette flows. The test case is two-dimensional and consists of a shear flow between two infinite flat plates: one is static and the other is moving in the 
$x$-direction at a constant velocity.

$$
U=M a \times c_{s, \infty}
$$

A shear force is transmitted to the fluid by the no-slip condition at the boundaries where thermal properties are given. Then, the temperature $T$ only depends on $y$. At steady state, the effects of viscous heat dissipation and thermal conduction balance out. During simulations, the heat capacity ratio at constant pressure $C_{p}$ and the Prandtl number $\operatorname{Pr}=$ $\left(\mu C_{p}\right) / \lambda$ are assumed to be constant.

For the benchmark, a simplified version of boundary conditions via cut cell approach 11 is adopted. Moreover, two specific thermal configurations are performed in a $2 \times 101 \times 1$ domain.

In the first one, the walls are at the same temperature such as $T_{\text {top wall }}=T_{\text {bottom wall }}=$ const. and the viscosity $\mu$ is constant. This configuration introduces a linear profile of $u_{x}$ velocity as a function of $y$ and the temperature can be theoretically expressed as $\frac{14}{4}$

$$
\frac{T}{T_{w}}=1+\frac{y}{H} \zeta\left(1-\frac{y}{H}\right)
$$

where $T_{w}$ is the temperature at the boundary walls, $H$ is the distance between them and $\zeta=\operatorname{Pr} \frac{\gamma-1}{2} \mathrm{Ma}^{2}$.

In the second configuration, the bottom wall is adiabatic with the Neumann temperature boundary condition $q_{y}=-\lambda(\partial T / \partial y)=0$ and the top wall is at constant temperature. For this configuration, the viscosity $\mu$ is updated with the help of a power law:

$$
\frac{\mu}{\mu_{0}}=\left(\frac{T}{T_{0}}\right)^{n} \quad \text { with } n=1
$$

Then, the velocity and temperature profiles are coupled and can be found analytically as 69 :

$$
\begin{aligned}
\frac{T}{T_{w}} & =1+\zeta\left[1-\left(\frac{u_{x}}{U}\right)^{2}\right] \\
\left(1+\frac{2}{3} \zeta\right) \frac{y}{H} & =\frac{u_{x}}{U}+\zeta\left[\frac{u_{x}}{U}-\frac{1}{3}\left(\frac{u_{x}}{U}\right)^{3}\right]
\end{aligned}
$$

The simulations are performed with the following conditions: the CFL varies between 0.23 and 0.77 to ensure the solution stability, the wall temperature is $T_{w}=300 \mathrm{~K}$, the initial 

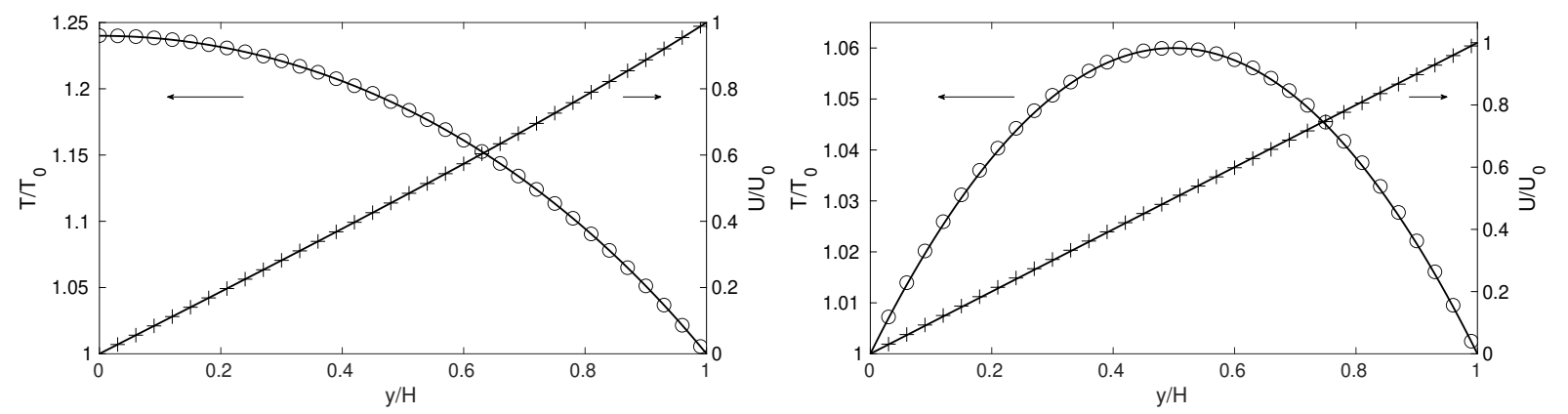

Figure 4: Temperature and velocity profiles of the thermal couette flow for $\gamma=7 / 5$, $\operatorname{Pr}=0.71$ and $\mathrm{Ma}=1.3$. The solid lines - correspond to analytical results; the numerical results + and o correspond respectively to the velocity profiles $U / U_{0}$ and the temperature profiles $T / T_{0}$. Boundary conditions: isothermal top wall and adiabatic bottom wall (left), isothermal walls (right).

pressure of the flow is $P_{0}=101325 \mathrm{~Pa}$ and $\sigma=0.7$. The two cases are tested through a wide range of supersonic parameters (Ma, Pr and $\gamma$ ). Fig. 4 shows the thermal configuration for a specific set of parameters $(\gamma=7 / 5, \operatorname{Pr}=0.71$ and $\mathrm{Ma}=1.3)$ while Table $\Pi$ summarizes all the results with the $\mathcal{L}_{2}$ error on the temperature defined as:

$$
\mathcal{L}_{2}^{T}=\frac{\sqrt{\sum_{i}\left(T_{i, \text { Simulation }}-T_{i, \text { Theory }}\right)^{2}}}{\sqrt{\sum_{i} T_{i, \text { Theory }}^{2}}}
$$

Note that, for this particular test case, the correction tensor $a_{\alpha \beta}^{F_{D}}$ (see Appendix A) cancels out because its components are either multiplied by a 0 velocity or derived with respect to a periodic direction in which macroscopic quantities are constant. Figure 4 and Table I show again a good agreement between simulations and analytical solutions, thus validating the viscous and thermal properties of the model.

Finally, a convergence study of the model is carried out by varying the grid resolution in the $y$-direction and measuring the $\mathcal{L}_{2}$ norm on the temperature. Figure 5 shows a second order convergence in space, classical of Lattice-Boltzmann models ${ }^{9}$. 
Table I: $\mathcal{L}_{2}$ Error Eq. (48) for different performed simulations.

\begin{tabular}{cccc}
\hline \hline \multicolumn{4}{c|}{ Isothermal bottom wall and Isothermal top wall } \\
\hline Mach Number & Prandtl Number & $\gamma$ & $\mathcal{L}_{2}^{T}$ Error \\
\hline 0.35 & 5 & $5 / 3$ & 0.0001499 \\
1 & 1.5 & $5 / 3$ & 0.0005695 \\
1.3 & 0.71 & $7 / 5$ & 0.0002404 \\
1.3 & 0.71 & $5 / 3$ & 0.0007031 \\
\hline \multicolumn{4}{c}{ Adiabatic bottom wall and Isothermal top wall } \\
\hline Mach Number & Prandtl Number & $\gamma$ & $\mathcal{L}_{2}^{T}$ Error \\
\hline 0.35 & 5 & $5 / 3$ & 0.0001605 \\
1 & 1.5 & $5 / 3$ & 0.0011649 \\
1.3 & 0.71 & $7 / 5$ & 0.0001973 \\
1.3 & 0.71 & $5 / 3$ & 0.0008085 \\
\hline \hline
\end{tabular}

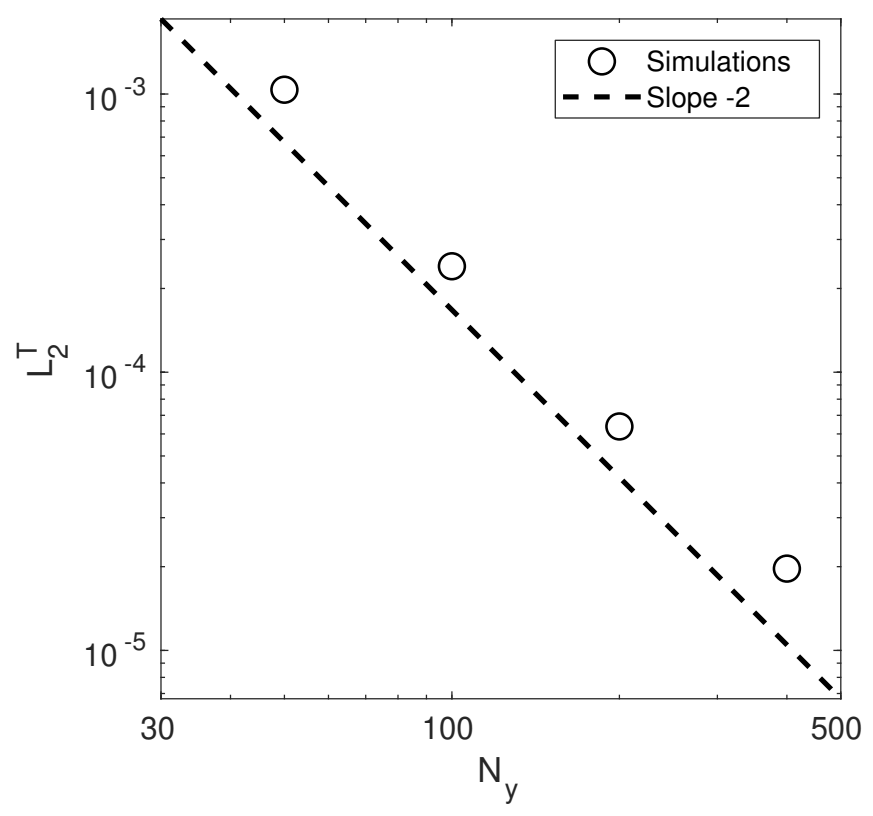

Figure 5: Convergence study : $\mathcal{L}_{2}^{T}$ norm Eq. (48) as a function of the resolution $N_{y}$. Carried out in the case of the isothermal top and bottom walls presented in Fig. 4.b. 

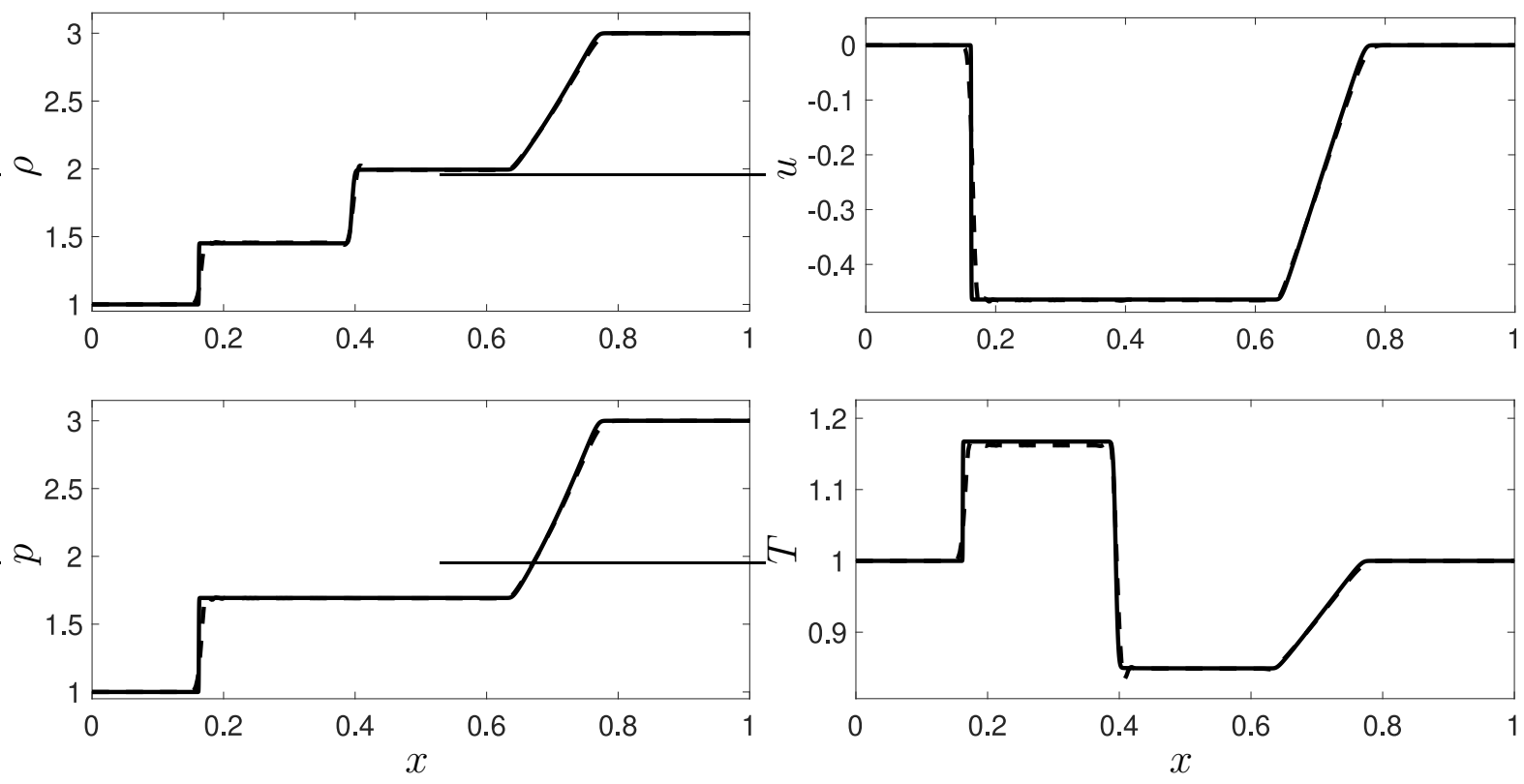

Figure 6: Sod shock tube solution. Density, velocity, pressure and temperature profiles as obtained with the HRR-p model (dashed) and reference (solid).

\section{E. One-dimensional shock tube}

Here the present model is assessed on a classical 1D Riemann problem. A Sod $\underline{70}$ shock tube is initialized with a left state $\left(\rho_{L}, u_{L}, p_{L}\right)=(1,0,1)$ and a right state $\left(\rho_{R}, u_{R}, p_{R}\right)=(3,0,3)$ in a domain of total length $L=1$ with an initial discontinuity located at $x=0.5$. This domain is discretized by 400 points, the time-step is related to the space increment through $\frac{\Delta t}{\Delta x}=0.2582$, the other parameters are $\gamma=1.4, \sigma=1, \mu=10^{-15}$. After 350 timesteps the solution is plotted in Fig. 6, showing from left to right a shock wave, a slip line and an expansion wave computed with the present model (dashed line). The reference solution was obtained with a classical first order HLLC $\underline{\underline{70}}$ (solid line) solver using with $10^{4}$ points. Agreement is very good: beside a small overshoot at the contact interface, the different levels are well captured.

\section{F. Shock-Vortex interaction}

The present model is finally assessed on an unsteady, viscous, compressible flow consisting of the interaction of a stationary shock wave with an isentropic vortex. The mean field is defined by the Mach number of the shock, $M_{s}$, and the left and right initial states are 
solution of the Rankine-Hugoniot problem,

$$
\begin{aligned}
& \frac{\rho_{R}}{\rho_{L}}=\frac{u_{L}}{u_{R}}=\frac{(\gamma+1) M_{s}^{2}}{(\gamma-1) M_{s}^{2}+2}, \\
& \frac{p_{R}}{p_{L}}=1+\frac{2 \gamma}{(\gamma+1)}\left(M_{s}^{2}-1\right) .
\end{aligned}
$$

Then, an isentropic vortex, as already defined in a previous validation, is superimposed in the unshocked region. The vortex will cross the shock and create a complex pattern of pressure waves that will be compared to a reference solution ${ }^{71}$. Physical parameters are set to $M_{s}=1.2, M_{v}=0.25, \gamma=1.4, R e=800, \operatorname{Pr}=0.75, p_{L}=1.0, T_{L}=1.0, u_{L}=M_{s} \sqrt{\gamma}$, which corresponds to "case $\mathrm{C}^{\prime \prime}$ in the reference solution ${ }^{71}$. The computational domain is $[0,28] \times[0,24]$, discretized by a $1120 \times 960$ mesh, shock and vortex positions are respectively $x_{s}=8$ and $\left(x_{c}, y_{c}\right)=(6,12)$. Numerical parameters were set to $C F L=0.87$ and $\sigma=0.7$ where the CFL was based on the unshocked region. Instantaneous density fields during the simulation are shown in Fig. 7.
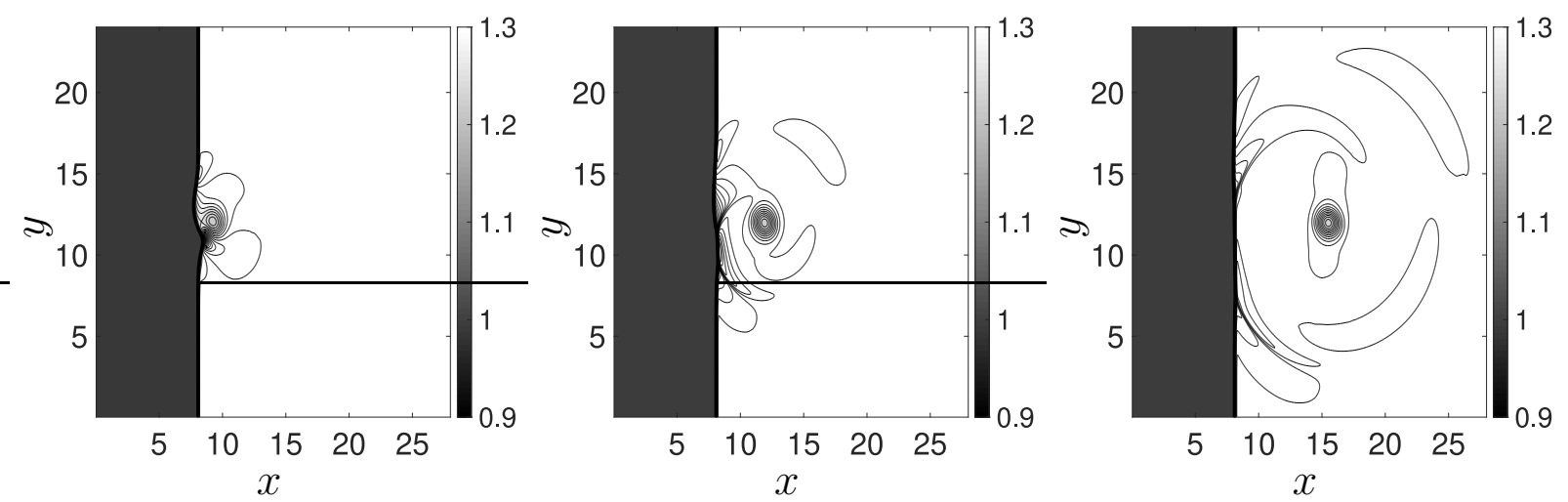

Figure 7: Density field for the shock-vortex interaction at $t=3 T$ (left), $t=6 T$ (center),

$$
t=10 T \text { (right). }
$$

Normalized pressure $\Delta p=\frac{p-p_{R}}{p_{R}}$ is then plotted on Fig. 8 along a radial cut of fixed angle $\theta=-45^{\circ}$ for $t=6 T, t=8 T$ and $t=10 T$ where we defined $T=\frac{R}{c_{R}}$ as the characteristic convective time of the vortex in the shocked region, showing very little difference with the reference 4 th/6th order time/spatial accuracy solution.

Precursor and second sound are also plotted on Fig. 9 and compared to ${ }^{71}$, showing again a good agreement. 


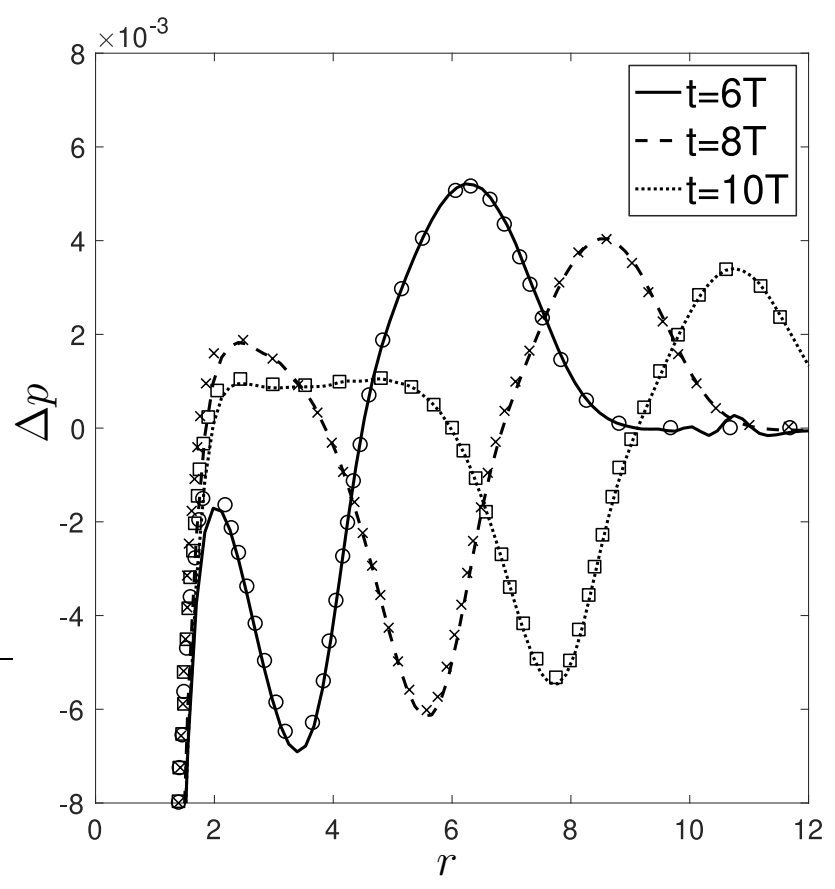

Figure 8: Radial cut at $\theta=-\pi / 4$ of the pressure variation $\Delta p$. Lines correspond to the present Lattice-Boltzmann solution and symbols denote the reference solution ${ }^{71}$.

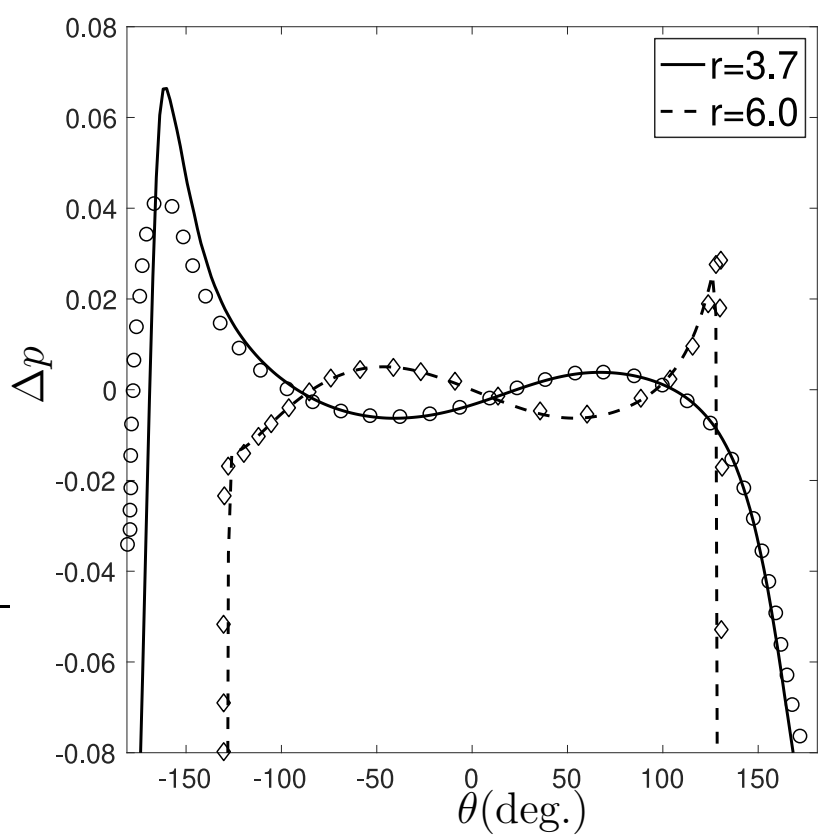

Figure 9: Circumferential pressure variation at $t=6 T$. Solid line corresponds to $r=3.7$ and dashed line to $r=6$. Markers refers to the corresponding reference solution ${ }^{71}$. 


\section{CONCLUDING REMARKS}

A new pressure-based hybrid regularized LBM, referred to as HRR- $p$ model, has been presented, for the simulation of compressible flows for Mach numbers ranging from 0 to 1.5. It is based on a fully explicit predictor-corrector segregated approach for the pressure, preserving the robustness of classical athermal LBM via the predictor step.

Through a variety of numerical experiments, we systematically validated (i) the Eulerian part of the system, via convection tests of the three natural Euler modes, (ii) the diffusive and viscous terms, through thermal Couette flows simulations, and (iii) the method's robustness and accuracy, through a 2D example of shock - vortex interactions.

A particularly interesting feature of the present model is that it is compatible with standard nearest-neighbor lattices (e.g. D3Q19), limiting the number of required scalars to a minimum (here, 19 density functions +1 entropy scalar field), making it a good candidate for future studies of 3D compressible flows. The computational cost of the method is of the same order as for classical athermal LBM, allowing to envision significant speedup for the simulation of compressible flows compared with conventional Navier-Stokes solvers. Future works include an extension to fully turbulent compressible flows.

\section{ACKNOWLEDGEMENTS}

Part of this research was supported by ANR, Renault, Airbus and SafranTech by the Industrial Chair Program ALBUMS (ANR-CHIND-18-ALBUMS). The French Space agency

(CNES) is acknowledged for supporting Song Zhao at M2P2. Centre de Calcul Intensif d'Aix-Marseille and GENCI-TGCC/CINES (Grant 2018-A0032A07679) are acknowledged for granting access to their high performance computing resources. We also acknowledge support from Labex MEC (ANR-10-LABX-0092) and the A*MIDEX project (ANR-11-IDEX0001-02), funded by the "Investissements d'Avenir".

\section{DATA AVAILABILITY}

The data that support the findings of this study are available from the corresponding author upon reasonable request. 


\section{REFERENCES}

${ }^{1} \mathrm{R}$. Löhner, "Towards overcoming the LES crisis," International Journal of Computational Fluid Dynamics , 1-11 (2019).

${ }^{2}$ Y. Feng, P. Sagaut, and W.-Q. Tao, "A compressible lattice boltzmann finite volume model for high subsonic and transonic flows on regular lattices," Computers \& Fluids 131, 45-55 (2016).

${ }^{3} \mathrm{C}$. Lin and K. H. Luo, "Mesoscopic simulation of nonequilibrium detonation with discrete boltzmann method," Combust. Flame (2018).

${ }^{4}$ Y. Feng, P. Boivin, J. Jacob, and P. Sagaut, "Hybrid recursive regularized lattice boltzmann simulation of humid air with application to meteorological flows," Physical Review E 100, 023304 (2019).

${ }^{5}$ S. Chen and G. D. Doolen, "Lattice boltzmann method for fluid flows," Annu. Rev. Fluid Mech. 30, 329-364 (1998).

${ }^{6} \mathrm{X}$. Shan, X.-F. Yuan, and H. Chen, "Kinetic theory representation of hydrodynamics: a way beyond the navier-stokes equation," J. Fluid Mech. 550, 413 (2006).

${ }^{7} \mathrm{X}$. Shan et al., "General solution of lattices for cartesian lattice bhatanagar-gross-krook models," Physical Review E 81, 036702 (2010).

${ }^{8}$ Q. Li, K. Luo, Q. Kang, Y. He, Q. Chen, and Q. Liu, "Lattice boltzmann methods for multiphase flow and phase-change heat transfer," Prog. Energy Combust. Sci. 52, 62-105 (2016).

${ }^{9}$ T. Krüger, H. Kusumaatmaja, A. Kuzmin, O. Shardt, G. Silva, and E. M. Viggen, The Lattice Boltzmann Method: Principles and Practice (Springer, 2016).

${ }^{10}$ Y. Feng, P. Boivin, J. Jacob, and P. Sagaut, "Hybrid recursive regularized thermal lattice boltzmann model for high subsonic compressible flows," J. Comput. Phys. 394, 82 - 99 (2019).

${ }^{11}$ Y. Feng, S. Guo, J. Jacob, and P. Sagaut, "Solid wall and open boundary conditions in hybrid recursive regularized lattice boltzmann method for compressible flows," Phys. Fluids 31, 126103 (2019).

${ }^{12}$ Y. Feng, M. Tayyab, and P. Boivin, "A lattice-boltzmann model for low-mach reactive flows," Combust. Flame 196, 249 - 254 (2018). 
${ }^{13}$ M. Tayyab, S. Zhao, Y. Feng, and P. Boivin, "Hybrid regularized lattice-boltzmann modelling of premixed and non-premixed combustion processes," Combust. Flame 211, 173-184 (2020).

${ }^{14}$ F. Renard, Y. Feng, J. Boussuge, and P. Sagaut, "Improved compressible hybrid lattice boltzmann method on standard lattice for subsonic and supersonic flows," arXiv preprint arXiv:2002.03644 (2020).

${ }^{15}$ J. Jacob, O. Malaspinas, and P. Sagaut, "A new hybrid recursive regularised bhatnagargross-krook collision model for lattice boltzmann method-based large eddy simulation," J. Turbul. , 1-26 (2018).

${ }^{16}$ T. Astoul, G. Wissocq, J. Boussuge, A. Sengissen, and P. Sagaut, "Non-hydrodynamic contributions in the spurious noise generated at grid refinement interfaces with the lattice boltzmann method," J. Comput. Phys., submitted (2020).

${ }^{17}$ G. Wissocq, P. Sagaut, and J.-F. Boussuge, "An extended spectral analysis of the lattice boltzmann method: Modal interactions and stability issues," J. Comput. Phys. 380, 311333 (2019).

${ }^{18}$ C. Coreixas, G. Wissocq, G. Puigt, J. F. Boussuge, and P. Sagaut, "Recursive regularization step for high-order lattice Boltzmann methods," Physical Review E 96, 1-22 (2017).

${ }^{19}$ C. Coreixas, B. Chopard, and J. Latt, "Comprehensive comparison of collision models in the lattice boltzmann framework: Theoretical investigations," Physical Review E 100, 033305 (2019).

${ }^{20}$ R. Zhang, X. He, and S. Chen, "Interface and surface tension in incompressible lattice boltzmann multiphase model," Comput. Phys. Commun. 129, 121-130 (2000).

${ }^{21}$ T. Lee and C.-L. Lin, "A stable discretization of the lattice boltzmann equation for simulation of incompressible two-phase flows at high density ratio," J. Comput. Phys. 206, 16-47 (2005).

${ }^{22} \mathrm{X}$. He, S. Chen, and R. Zhang, "A lattice boltzmann scheme for incompressible multiphase flow and its application in simulation of rayleigh-taylor instability," J. Comput. Phys. 152, 642-663 (1999).

${ }^{23}$ R. Zhang, X. He, G. Doolen, and S. Chen, "Surface tension effects on two-dimensional two-phase kelvin-helmholtz instabilities," Adv. Water Resour. 24, 461-478 (2001). 
${ }^{24}$ X. He, R. Zhang, S. Chen, and G. Doolen, "On the three-dimensional rayleigh-taylor instability," Phys. Fluids 11, 1143-1152 (1999).

${ }^{25}$ Y. Cao, "Variable property-based lattice boltzmann flux solver for thermal flows in the low mach limit," Int. J. Heat Mass Transfer 101, 254-264 (2016).

${ }^{26}$ O. Filippova and D. Hänel, "A novel lattice bgk approach for low mach number combustion," J. Comput. Phys. 158, 139-160 (2000).

${ }^{27}$ O. Filippova and D. Haenel, "A novel numerical scheme for reactive flows at low mach numbers," Computers Physics Communications 129, 267-274 (2000).

${ }^{28}$ M. Ashna and M. Rahimian, "Lbm simulation of head-on collision of evaporating and burning droplets in coalescence regime," Int. J. Heat Mass Transfer 109, 520-536 (2017).

${ }^{29}$ T. Lee, C.-L. Lin, and L.-D. Chen, "A lattice boltzmann algorithm for calculation of the laminar jet diffusion flame," J. Comput. Phys. 215, 133-152 (2006).

${ }^{30}$ T. Inamuro, T. Ogata, S. Tajima, and N. Konishi, "A lattice boltzmann method for incompressible two-phase flows with large density differences," J. Comput. Phys. 198, 628-644 (2004).

${ }^{31}$ S. Mukherjee and J. Abrahams, "A pressure-evolution-based multi-relaxation-time highdensity-ratio two-phase lattice-boltzmann model," Computers and Fluids 36, 1149-1158 (2007).

${ }^{32}$ K. Connington, M. Miskin, T. Lee, H. Jaeger, and J. Morris, "Lattice-boltzmann simulations of particle-laden liquid bridges: effect of volume fraction and wettability," International Journal of Multiphase Flows 76, 32-46 (2015).

${ }^{33} \mathrm{~T}$. Lee, "Effects of incompressibility on the elimination of parasitic currents in lattice boltzmann equation method for binary fluids," Computers and Mathematics with Applications 58, 987-994 (2009).

${ }^{34}$ A. Fakhari, M. Geier, and T. Lee, "A mass-conserving lattice boltzmann method with dynamic grid refinement for immiscible two-phase flows," J. Comput. Phys. 315, 434-457 (2016).

${ }^{35}$ K. Sahu and S. Vanka, "A multiphase lattice boltzmann study of buoyancy-induced mixing in a tilted channel," Computers and Fluids 50, 199-215 (2011).

${ }^{36} \mathrm{~K}$. Connington and T. Lee, "Lattice boltzmann simulations of forced wetting transitions of drops on superhydrophobic surfaces," J. Comput. Phys. 250, 601-615 (2013). 
${ }^{37}$ T. Lee and L. Liu, "Lattice boltzmann simulations of micron-scale drop impact on dry surfaces," J. Comput. Phys. 229, 8045-8063 (2010).

${ }^{38}$ L. Amaya-Bower and T. Lee, "Single bubble rising dynamics for moderate reynolds number using lattice boltzmann method," Computers and Fluids 39, 1191-1207 (2010).

${ }^{39}$ R. Abadi and M. Rahimian, "hybrid lattice boltzmann finite difference model for simulation of phase change in a ternary fluid," Int. J. Heat Mass Transfer 127, 704-716 (2018).

${ }^{40}$ E. Reyhanian, M. Rahimian, and S. Farsid Chini, "Investigation of 2d drop evaporation on a smooth and homogeneous surface using lattice boltzmann method," Int. Commun. Heat Mass Transfer 89, 64-72 (2018).

${ }^{41}$ H. Safari, M. Rahimian, and M. Krafczyk, "Extended lattice boltzmann method for simulation of thermal phase change in two-phase fluid flow," Physical Review E 88, 013304 (2013).

${ }^{42}$ H. Safari, M. Rahimian, and M. Krafczyk, "Consistent simulation of droplet evaporation based on phase-field multiphase lattice boltzmann method," Physical Review E 90, 033305 (2014).

${ }^{43}$ M. Mohammadi-Shad and T. Lee, "Phase-field lattice boltzmann modelling of boiling using a sharp-interface energy solver," Physical Review E 96, 013306 (2017).

${ }^{44} \mathrm{R}$. Knikker, "A comparative study of high-order variable-property segregated algorithms for unsteady low mach number flows," Int. J. Numer. Methods Fluids 66, 303-427 (2011).

${ }^{45}$ N. Kwatra, J. Su, J. Gretarsson, and R. Fedkiw, "A method for avoiding the acoustic time step restriction in compressible flow," J. Comput. Phys. 228, 4146-4162 (2009).

${ }^{46}$ N. Nerinckx, J. Vierendeels, and E. Dick, "Mach-uniformity through the coupled pressure and temperature correction term," J. Comput. Phys. 206, 597-623 (2005).

${ }^{47}$ G. Hauke, A. Landaberea, G. Inaki, and J. Canales, "A segregated method for compressible flow computation. part i: Isothermal compressible flows," Int. J. Numer. Methods Fluids 47, 271-323 (2005).

${ }^{48}$ G. Hauke, A. Landaberea, G. Inaki, and J. Canales, "A segregated method for compressible flow computation. part ii: General divariant compressible flows," Int. J. Numer. Methods Fluids 49, 183-209 (2005).

${ }^{49}$ M. Darwish, F. Moukalled, and B. Sekar, "A unified formulation of the segregated class of algorithms for multifluid flow at all speeds," Numerical Heat Transfer, Part B: Fundamentals 40, 99-137 (2013). 
${ }^{50}$ F. Moukalled and M. Darwish, "Pressure-based algorithms for multifluid flow at all speeds. part i: Mass conservation formulation," Numerical Heat Transfer, Part B: Fundamentals 45, 495-522 (2004).

${ }^{51}$ A. Chorin, "A numerical method for solving incompressible viscous flow problems," J. Comput. Phys. 2, 12-26 (1967).

${ }^{52}$ A. Chorin, "Numerical solution of the navier-stokes equations," Mathematics of Computations 22, 745-762 (1968).

${ }^{53}$ C. Hirt and B. Nichols, "Adding limited compressibility to incompressible hydrocodes," J. Comput. Phys. 34, 390-400 (1980).

${ }^{54}$ P. Madsen and H. Schäffer, "A discussion of artificial compressibility," Coastal Eng. 53, 93-98 (2006).

${ }^{55}$ W. Kim and S. Menon, "An unsteady incompressible navier-stokes solver for large-eddy simulation of turbulent flows," Int. J. Numer. Methods Fluids 31, 983-1017 (1999).

${ }^{56}$ A. Kajzer and J. Pozorski, "Application of entropically damped artificial compressibility model to direct numerical simulation of turbulent channel flow," Computers and Mathematics with Applications 76, 997-1013 (2018).

${ }^{57}$ A. Toutant, "Numerical simulations of unsteady viscous incompressible flows using general pressure equation," J. Comput. Phys. 374, 822-842 (2018).

${ }^{58}$ X. He, G. D. Doolen, and T. Clark, "Comparison of the lattice boltzmann method and the artificial compressibility method for navier-stokes equations," J. Comput. Phys. 179, $439-451(2002)$.

${ }^{59}$ P. Asinari, T. Ohwada, E. Chiavazzo, and A. Di Rienzo, "Link-wise artificial compressibility method," J. Comput. Phys. 231, 5109-5143 (2012).

${ }^{60}$ T. Lee and C.-L. Lin, "Pressure evolution lattice-boltzmann-equation method for two-phase flow with phase change," Physical Review E 67, 056703 (2003).

${ }^{61}$ S. Karni, "Hybrid multifluid algorithms," SIAM Journal of Scientific Computations 17, 1019-1039 (1996).

${ }^{62}$ R. Abgrall and S. Karni, "Computations of compressible multifluids," J. Comput. Phys. 169, 594-623 (2001).

${ }^{63}$ A. Toutant, "General and exact pressure evolution equation," Physics Letter A 381, 3739$3742(2017)$. 
${ }^{64}$ J. Jacob and P. Sagaut, "Wind comfort assessment by means of large eddy simulation with lattice boltzmann method in full scale city area," Build. Environ. 139, 110-124 (2018).

${ }^{65}$ P. J. Dellar, "An interpretation and derivation of the lattice boltzmann method using strang splitting," Computers \& Mathematics with Applications 65, 129-141 (2013).

${ }^{66}$ Y.-L. Feng, S.-L. Guo, W.-Q. Tao, and P. Sagaut, "Regularized thermal lattice boltzmann method for natural convection with large temperature differences," Int. J. Heat Mass Transfer 125, 1379-1391 (2018).

${ }^{67}$ S. Marié, D. Ricot, and P. Sagaut, "Comparison between lattice boltzmann method and navier-stokes high order schemes for computational aeroacoustics," J. Comput. Phys. 228, 1056-1070 (2009).

${ }^{68}$ D. Fabre, L. Jacquin, and J. Sesterhenn, "Linear interaction of a cylindrical entropy spot with a shock," Physics of Fluids 13, 2403-2422 (2001).

${ }^{69}$ H. W. Liepmann and A. Roshko, Elements of gasdynamics (Courier Corporation, 2001).

${ }^{70}$ E. F. Toro, Riemann solvers and numerical methods for fluid dynamics: a practical introduction (Springer Science \& Business Media, 2009).

${ }^{71} \mathrm{O}$. Inoue and Y. Hattori, "Sound generation by shock-vortex interactions," J. Fluid Mech. 380, 81-116 (1999).

\section{Appendix A: Forcing terms}

Depending on the order of the LBM in the Hermite space, a different forcing term should be added to achieve a viscous stress tensor

$$
a_{\alpha \beta}^{\mathrm{neq}}=-\mu\left(u_{\alpha, \beta}+u_{\beta, \alpha}-\frac{2}{3} u_{\gamma, \gamma} \delta_{\alpha \beta}\right) .
$$

For the $D 3 Q 19 r$ base, the projected forcing term reads as

$$
\begin{aligned}
a_{\alpha \beta}^{F^{E}} & =c_{s}^{2} u_{\alpha}[\rho(1-\theta)]_{, \beta}+c_{s}^{2} u_{\beta}[\rho(1-\theta)]_{, \alpha} \\
& +\delta_{\alpha \beta} \rho c_{s}^{2} \frac{2}{3} u_{\gamma, \gamma}-a_{\alpha \beta}^{\mathrm{cor}}+a_{\alpha \beta}^{F_{D}}
\end{aligned}
$$

where we respectively define $a_{\alpha \beta}^{\text {cor }}$, a correction tensor due to the deflection of second order moments of the population introduced by the modification of the mass equation, which can be evaluated as

$$
\begin{gathered}
a_{\alpha \beta}^{\text {cor }} \equiv c_{s}^{2} \delta_{\alpha \beta}\{\rho(t+\Delta t, \boldsymbol{x})[1-\theta(t+\Delta t, \boldsymbol{x})] \\
-\quad \rho(t, \boldsymbol{x})[1-\theta(t, \boldsymbol{x})]\}
\end{gathered}
$$


and $a_{\alpha \beta}^{F_{D}}$ the correction tensor due to the defect of the lattice at third order

$$
a_{\alpha \beta}^{F_{D}}=-\left(\begin{array}{ccc}
\left(\rho u_{x}^{3}\right)_{, x} & \left(\rho u_{x} u_{y} u_{z}\right)_{, z} & \left(\rho u_{x} u_{y} u_{z}\right)_{, y} \\
\left(\rho u_{x} u_{y} u_{z}\right)_{, z} & \left(\rho u_{y}^{3}\right)_{, y} & \left(\rho u_{x} u_{y} u_{z}\right)_{, x} \\
\left(\rho u_{x} u_{y} u_{z}\right)_{, y} & \left(\rho u_{x} u_{y} u_{z}\right)_{, x} & \left(\rho u_{z}^{3}\right)_{, z}
\end{array}\right)
$$

where all the differential operations are performed using first order upwind FD. The final expression of the forcing term is then

$$
F_{i}^{E}=\frac{\omega_{i}}{2 c_{s}^{4}} \mathcal{H}_{i, \alpha \beta}^{(2)} a_{\alpha \beta}^{F^{E}}
$$

\section{Appendix B: Recursive reconstruction of third order off-equilibrium tensor}

The third order non-equilibrium tensor is achieved via a recursive procedure from second order tensor, and reads as

$$
\begin{aligned}
& a_{\alpha \beta \gamma}^{(3), \text { neq }}=u_{\alpha} \tilde{a}_{\beta \gamma}^{\text {neq }}+u_{\beta} \tilde{a}_{\alpha \gamma}^{\text {neq }}+u_{\gamma} \tilde{a}_{\alpha \beta}^{\text {neq }} \\
& a_{1}^{(3 r), \text { neq }} \equiv a_{x x y}^{(3), \text { neq }}+a_{y z z}^{(3), \text { neq }} \\
& a_{2}^{(3 r), \text { neq }} \equiv a_{x z z}^{(3) \text {,neq }}+a_{x y y}^{(3), \text { neq }} \\
& a_{3}^{(3 r), \text { neq }} \equiv a_{y y z}^{(3) \text {,neq }}+a_{x x z}^{(3), \text { neq }} \\
& a_{4}^{(3 r), \text { neq }} \equiv a_{x x y}^{(3) \text {,neq }}-a_{y z z}^{(3), \text { neq }} \\
& a_{5}^{(3 r), \text { neq }} \equiv a_{x z z}^{(3) \text {,neq }}-a_{x y y}^{(3), \text { neq }} \\
& a_{6}^{(3 r), \text { neq }} \equiv a_{y y z}^{(3) \text { neq }}-a_{x x z}^{(3), \text { neq }}
\end{aligned}
$$

\section{Appendix C: MUSCL-Hancock method}

Let's consider the following 1D transport equation

$$
\frac{s_{i}^{n+1}-s_{i}^{n}}{\Delta t}+\frac{F\left(s_{i+\frac{1}{2}}^{n}\right)-F\left(s_{i-\frac{1}{2}}^{n}\right)}{\Delta x}=0 .
$$

MUSCL-Hancock intercell fluxes $F\left(s_{i+\frac{1}{2}}^{n}\right)$ and $F\left(s_{i-\frac{1}{2}}^{n}\right)$ for a non-conservative form are computed as follows :

i) Data reconstruction. Evaluate the extrapolated intercell values at the left and right sides of the $i$ th cell as

$$
s_{i, L}=s_{i}-\frac{\Delta x}{2} \phi_{i} \Delta_{i}, \quad s_{i, R}=s_{i}+\frac{\Delta x}{2} \phi_{i} \Delta_{i}
$$


with $\Delta_{i}$ the local approximated slope

$$
\Delta_{i}=\frac{1}{2}\left[(1+\kappa)\left(s_{i}-s_{i-1}\right)+(1-\kappa)\left(s_{i+1}-s_{i}\right)\right]
$$

and $\phi_{i}$ a Van Albada flux limiter, only used for shocked flows, which reads as

$$
\phi_{i}=\frac{r(r+1)}{1+r^{2}}, \quad r=\frac{s_{i}-s_{i-1}}{s_{i+1}-s_{i}} .
$$

In order to improve the accuracy, it was chosen to evaluate dynamically $\kappa=$ $\frac{1}{3}\left[\frac{2 \Delta t u_{i}}{\Delta x}-\operatorname{sign}\left(u_{i}\right)\right]$, leading to a third-order accurate scheme in both space and time for a constant $u_{i}$ value $\mathrm{e}^{70}$.

ii) Data evolution. Let the extrapolated boundary values evolve of a time $\frac{\Delta t}{2}$ according to

$$
\begin{aligned}
& \bar{s}_{i, L}=s_{i, L}+\frac{\Delta t u_{i}}{2 \Delta x}\left(s_{i, L}-s_{i, R}\right), \\
& \bar{s}_{i, R}=s_{i, R}+\frac{\Delta t u_{i}}{2 \Delta x}\left(s_{i, L}-s_{i, R}\right) .
\end{aligned}
$$

iii) Solution of the piece-wise constant problem. Finally we simply compute $F\left(s_{i+\frac{1}{2}}^{n}\right)$ and $F\left(s_{i-\frac{1}{2}}^{n}\right)$ using :

$$
\begin{aligned}
& F\left(s_{i+\frac{1}{2}}^{n}\right)= \begin{cases}u_{i} \bar{s}_{i, R} & \text { if } u_{i} \geq 0 \\
u_{i} \bar{s}_{i+1, L} & \text { if } u_{i}<0\end{cases} \\
& F\left(s_{i-\frac{1}{2}}^{n}\right)= \begin{cases}u_{i} \bar{s}_{i-1, R} & \text { if } u_{i} \geq 0 \\
u_{i} \bar{s}_{i, L} & \text { if } u_{i}<0\end{cases}
\end{aligned}
$$

Note that this scheme uses a 5-points stencil because the evaluation of the slope is non-local. 